\title{
Confrontación generacional y cuestiones de género: una lucha activa dentro y fuera de las escuelas uruguayas
}

\author{
Generational confrontation and gender issues: An active \\ struggle within and outside schools in Uruguay
}

DOI: https://doi.org/10.32870/dse.v0i21.654

\section{Gonzalo Iván Gelpi*}

\begin{abstract}
Resumen
En este artículo se reflexiona sobre el rol que tienen actualmente las cuestiones vinculadas al género en los procesos de confrontación generacional entre los adolescentes y sus adultos referentes en el marco de la construcción de sus identidades, así como también se aborda la politización de la sexualidad con un enfoque de derechos humanos, a partir de la militancia activa de los estudiantes dentro de sus propias escuelas en el nivel de enseñanza media. Para cumplir con el objetivo, se presenta una breve historización del sistema educativo formal en Uruguay, se ahonda en las características de la educación sexual en nuestro país y se retoman conceptualizaciones teóricas sobre la adolescencia en tanto etapa del ciclo de vida. El marco teórico empleado para la elaboración de este escrito es predominantemente multidisciplinario, destacándose la articulación entre autores y autoras del campo de las ciencias de la educación, de las ciencias sociales y de las ciencias de la salud. Asimismo, mencionar que la idea de este trabajo surge con base en: a) la experiencia clínica con población adolescente; $b$ ) investigaciones sociales coordinadas en el ámbito educativo sobre género, sexualidad y diversidad sexual en la ciudad de Montevideo; y c) actividades de extensión sobre distintos temas que integran la agenda de género, ejecutadas en instituciones educativas de Enseñanza Media en la ciudad antes mencionada, con población adolescente y docente.
\end{abstract}

Palabras clave: educación sexual - enseñanza media - adolescencia - género - ciudadanía.

\begin{abstract}
This article reflects on the role that gender issues currently have in the processes of generational confrontation between adolescents and their adult referents in the framework of the construction of their identities, as well as the politicization of sexuality with a human rights approach based on the active militancy of the students within their own schools at the level of Secondary Education. To this aim, the paper presents a brief historical presentation of the formal education system in Uruguay, explores the characteristics of
\end{abstract}

* Maestro en Género, Sociedad y Políticas Públicas. Docente del Programa Género, Sexualidades y Salud Reproductiva, Universidad de la República (UdelaR) y del Programa de Posgrado en Género, Sociedad y Políticas Públicas, Flacso-Argentina. Líneas de investigación: estudios de género y diversidad sexual focalizados en población adolescente y generalmente aplicados en el campo de la educación. Co-coordinador del Centro de Referencia Amigable (CRAm), Uruguay. ggelpi@psico.edu.uy 
sex education in our country and theoretical conceptualizations about adolescence are approached as a stage in the life cycle. The theoretical framework adopted for this paper is predominantly multidisciplinary, highlighting the articulation between authors in the field of education sciences, social sciences and health sciences. The idea for this work arose from clinical experience with adolescent population, social research coordinated in the field of education on gender, sexuality and sexual diversity in the city of Montevideo, and outreach activities on the different topics that make up the gender agenda conducted with teenagers and teachers in secondary education institutions in Montevideo.

Keywords: sex education - secondary education - adolescence - gender - citizenship.

\section{Introducción}

\section{El sistema educativo formal en Uruguay: Una breve historización}

En el año 1874 José Pedro Varela escribió La educación del pueblo. Esta obra ofició de piedra fundamental, no solo de la escuela primaria sino de toda la educación en Uruguay. Se trató de una obra de avanzada, reflejo de un ideario liberal y republicano que lo guiaba, recogiendo la experiencia y elaboración en materia educativa de las democracias más adelantadas del mundo. El sistema educativo uruguayo formal, finalmente se sustentaría en el modelo francés (Errandonea, 2014). Barrán (1990) señala a la Ley de Educación Común de 1877 como un hito histórico puesto que fue vanguardista en la región. La obligatoriedad de la enseñanza primaria también inauguró una etapa caracterizada por el disciplinamiento. "La implementación de la escuela pública, laica, gratuita y obligatoria imprimió la obediencia y el estudio" (Barrán, 1990: 21).

El discurso vareliano también era promotor de la educación de las mujeres, pero de una educación rudimentaria, reducida al aprendizaje impreciso de lectura, escritura, cuentas y ciertas labores manuales. Las mujeres pertenecientes a sectores altos de la sociedad podían acceder a una educación más integral, mas nunca pensada para el ejercicio de ciudadanía (Errandonea, 2014). En este sentido, en un comienzo, las escuelas eran mixtas hasta los ocho años. Luego de terminada la primaria, las familias usualmente decidían no enviar a las mujeres a secundaria a pesar de que legalmente era posible, por lo que nunca hubo una prohibición legal sino más bien impedimentos culturales que no permitían que varones y mujeres compartieran las escuelas secundarias, porque los modos de pensamiento y conocimiento eran entendidos como simbólicamente masculinos (Errandonea, 2014).

Desde la perspectiva de Errandonea, el modelo propuesto reprimía fuertemente las manifestaciones de lo sexual y encauzaba la agresividad y la violencia con el objetivo de promover el "progreso económico". De este modo, los educadores (maestros/as, profesoras/es) —en tanto actores sociales y educativos - cumplían un rol fundamental, idealizando valores como el trabajo, la disciplina, la puntualidad, el orden y la higiene. 
Posteriormente, la Ley de 1865 definió que los establecimientos de enseñanza secundaria pasaran a denominarse Liceos (Nahum, 2008). Luego, la Ley Universitaria de 1885 otorgó una doble función a la enseñanza secundaria: la ampliación de la enseñanza primaria y la preparación para estudios superiores, lo que generó una tensión entre una educación que brindase una cultura general no especializada (que tuviera como objetivo formar para una vida social integral, las responsabilidades ciudadanas y familiares) y la que exigía una preparación exigente para los estudios superiores (Silveira, 2015). Más tarde, en los primeros quince años del siglo XX, bajo los mandatos presidenciales de José Batlle y Ordoñez, se crearon liceos en todos los departamentos porque desde la perspectiva del movimiento batllista, fomentar la educación y la ciudadanía era el mejor modo de fortalecer la democracia (Barrán, 1990). En este periodo la matrícula femenina aumentó significativamente.

De todos modos, se debe señalar que el lema batllista "Todos somos Iguales" presenta limitaciones porque, pese a sus buenas intenciones, dicho lema invisibilizó las diferencias y cooperó para instaurar también en el ámbito educativo una cultura de la homogeneidad. En periodos caracterizados por aires nacionalistas, donde la identidad nacional se continuaba forjando, no se aprovechó la oportunidad de valorar la diversidad, a sabiendas de que Uruguay fue un país receptor de inmigrantes, que produjo en nuestro territorio el encuentro entre sujetos con distintas lenguas, costumbres y cosmovisiones (Barrán, 1990).

La Educación Media comenzó a democratizarse recién en los años cincuenta, pero hasta la segunda mitad de los años ochenta no accedieron al sistema adolescentes provenientes de sectores en situación de vulnerabilidad social, asistiendo a los centros una población bastante homogénea (Viscardi, 2003). Asimismo, se debe resaltar que durante la dictadura militar (1973-1984), los dispositivos pedagógicos y las estructuras institucionales sufrieron cambios que desembocaron en lo que algunos autores denominaron "deterioro educacional" (Bayce, 1987). El régimen dictatorial se caracterizó, entre muchos otros horrores, por perseguir, violentar y desaparecer a sujetos y cuerpos disidentes sexuales. En los edificios escolares se robustecieron los dispositivos de disciplinamiento en materia de género y sexualidad, reforzando en todo momento los modelos hegemónicos de masculinidad y feminidad, así como también el orden de género tradicional (Sempol y Graña, 2012). En ese mismo contexto se produjo la expansión de la educación privada, generándose paulatinamente una mayor fragmentación social.

De hecho, en la actualidad, aunque existen matices, se puede afirmar que las trayectorias educativas de quienes asisten a educación pública y privada son diferentes y son atravesadas —especialmente en el caso de la educación pública - por desigualdades sociales que cooperan con la producción de exclusión social (Filgueira y Kaztman, 2001). Más adelante, a partir del año 1995, con vaivenes, el país inició un proceso de Reforma Educativa denominada Plan Rama (Freitas, 2016). Posteriormente, ya iniciado el siglo XXI, con el advenimiento de los gobiernos progresistas, se pensó en programas (en educación primaria y secundaria) que inauguraron 
nuevos espacios informales y dispositivos pedagógicos con el objetivo de asegurarse la permanencia de los estudiantes en el sistema educativo formal y de intentar reinsertar a quienes ya se habían desvinculado.

Al presente, la estructura del sistema educativo uruguayo puede ser definida como piramidal y centralizada en un Consejo Directivo Central (CODICEN), del cual se derivan los cuatro consejos: Consejo de Educación Primaria (CEP) que incluye Educación Inicial; Consejo de Educación Secundaria (CES); Consejo de Educación Técnica Profesional (CETP), que incluye a la Universidad del Trabajo del Uruguay (UTU); y Consejo de Formación Docente (CFE) (Silveira, 2015). A su vez, el sistema nacional de educación se divide en dos grandes etapas, una correspondiente a la Educación Primaria - que incluye el preescolar - y otra a la Educación Media, que se divide en Educación Secundaria y Educación Técnica. Dicho sistema históricamente ha tenido a la clase media como eje articulador de la expresión de expectativas y necesidades (Viscardi, 2008).

Ya con esa estructura, en el año 2006 la educación extendió los niveles de obligatoriedad, incluyendo a la enseñanza preescolar (cuatro y cinco años) y a la Educación Media (Ciclo Básico Obligatorio). Posteriormente, en el año 2009, se aprobó la Ley General de Educación Núm. 18.437. En el escrito se proclama a la educación como un derecho humano fundamental y se afirma que: "el Estado garantizará y promoverá una educación de calidad para todos sus habitantes, a lo largo de toda la vida, facilitando la continuidad educativa (Ley Núm. 18.437, 2009). El Art. 2 sostiene: "reconócese el goce y el ejercicio del derecho a la educación, como un bien público y social [...] de todas las personas sin discriminación alguna" (Ley Núm. 18.437, 2009). Mientras que, en el Art. 8, se manifiesta: "el Estado asegurará los derechos de aquellos colectivos minoritarios o en especial situación de vulnerabilidad, con el fin de asegurar la igualdad de oportunidades en el pleno ejercicio del derecho a la educación y su efectiva inclusión social" (Ley Núm. 18.437, 2009).

\section{La educación sexual en el sistema educativo formal uruguayo: Diseño, implementación, evaluación y desafíos contemporáneos}

En la presente Ley, también se incluye a la educación sexual como un eje trasversal a todos los niveles, afirmando que, "tendrá como propósito proporcionar instrumentos adecuados que promuevan en educadores y educandos, la reflexión crítica ante las relaciones de género y la sexualidad en general para un disfrute responsable de la misma" (Ley Núm. 18.437, 2009). Es importante hacer visible que la escuela, desde su surgimiento hasta la institucionalización de dicho programa, siempre educó sexualmente al estudiantado con diferentes métodos y objetivos dependiendo de la época. En un comienzo, incluso la educación sexual fue una pedagogía de producción de normalidad (Britzman, 1996).

En Uruguay, entre los años 1920 y 1940, se discutió sistemáticamente sobre qué contenidos integran la denominada educación sexual, qué forma se le da a esta y cuál es el mejor modo 
para enseñarla; una referente del tema durante la época fue la feminista Paulina Luisi (Darré, 2005). Según Darré (2005), probablemente su institucionalización se dilató debido a resistencias desde la Iglesia católica, por los diversos sentidos y significados que los sujetos le otorgan a la educación sexual, porque históricamente se ha legitimado más a los médicos que a los profesores para hablar sobre sexualidad y porque dicha educación rompe el principio básico del discurso pedagógico sobre la transmisión de saberes construidos como verdades, ya que en materia de sexualidad todo es muy subjetivo. Se puede sostener, además, que la educación sexual ha sido y es uno de los campos donde múltiples discursos intervienen en una disputa política de la sexualidad. Esto se debe, en parte, a que la educación sexual "constituye un espacio situado en la convergencia de diferentes dimensiones que lo hacen atravesar tanto el campo educativo como el de la salud y el de las políticas demográficas y sanitarias" (Darré, 2005: 27). Así, la educación sexual aparece como una práctica social que ha constituido un campo de posibilidades donde se articulan y enfrentan discursos sociales tales como el discurso científico, el pedagógico y el jurídico.

Asimismo, Foucault (1998) señala que la "sexualidad adolescente" es producida por la combinación de diferentes tecnologías que parten de una visión patologizadora (deseos irrefrenables, erotismo sin medir consecuencias, onanismo, etc.) que debe encauzarse y disciplinarse. El ingreso al espacio educativo de los discursos sobre la sexualidad se realizó reproduciendo visiones higienistas, biologicistas y medicalizadas, que buscaban hacer énfasis en una perspectiva clínica que promovía la minimización de los daños (infecciones de transmisión sexual) y la disociación de esta dimensión con la subjetividad de los adolescentes.

Sin embargo, con este nuevo marco legal, en el año 2009 la Administración Nacional de Educación Pública (ANEP) creó el Programa de Educación Sexual (PES) con el objetivo de que fuese una herramienta para cumplir con los compromisos asumidos en el escenario políticoinstitucional. Se destaca como aspecto clave que la filosofía inspiradora de la educación sexual en Uruguay fue consensuada entre los diferentes partidos políticos con representación parlamentaria, lo que constituye una fortaleza para la puesta en agenda y la transversalización de la temática (UNFPA, 2014).

En el nivel de Enseñanza Media, se pensó en la figura de un profesor referente de sexualidad por centro. Dicha figura trabaja en forma transversal, con docentes de las diferentes asignaturas en cada centro liceal, además de hacerlo con madres, padres o representantes de adolescentes y en talleres específicos con estudiantes. Los Referentes se encuentran ordenados en listas departamentales y cumplen diez horas semanales de labor por cada Liceo (Abero, 2015). Quienes poseen el cargo, suelen ser profesores de biología y formación ciudadana, reproduciendo generalmente los "modelos dominantes" de tradición de la educación sexual (Morgade, 2006). Con la naciente institucionalización de la educación sexual en el sistema educativo uruguayo formal, se previó la inclusión de contenidos en diferentes asignaturas y niveles (Abero, 2015), la 
pregunta es: ¿eso es suficiente? ¿es posible encuadrar la educación sexual? ¿qué tipo de educación sexual está disponible? ¿hay criterios unánimes en torno a la trasmisión de saberes en este campo?

A partir de todo lo antes expuesto sobre la vigente ley, es válido interrogarse si en la práctica efectivamente todos los niños, niñas y adolescentes pueden ejercer dicho derecho, ¿qué obstáculos se los impide en lo cotidiano? ¿qué valores defienden las instituciones educativas en materia de sexualidad? ¿desde qué paradigmas se interviene? ¿se promueve la diversidad? ¿de qué modo? ¿hay inclusión? ¿qué se incluye? ¿desde dónde? ¿qué mensajes de sexualidad se difunden en los centros? ¿de qué tipo de sexualidades se habla? ¿cómo se educa en sexualidad? ¿cómo circula el género por los edificios escolares? ¿cómo se lo construye desde la propia escuela? ¿qué sucede cuando se hace visible el (des)orden genérico?

En esta línea, pese a los esfuerzos realizados, es importante reconocer que aún existen problemas estructurales de la escuela que afectan a la población estudiantil y, en ocasiones, a los propios docentes, como por ejemplo: la heteronormatividad institucional, el sexismo, los mandatos de género, las normas de género promovidas y la respectiva vigilancia de género aún persistente, el racismo, la homo-lesbo-transfobia y el clasismo latente (Louro, 2000; Marrero, 2003; Morgade, 2006; Marrero, Mallada y Cafferatta, 2008; Elizalde, 2009; Marrero y Mallada, 2009; Junqueira, 2013; Sempol, 2013; Schenck, 2014; Ovejas Negras y GLSEN, 2016; Gelpi, 2019).

Volviendo al tema en cuestión, durante el año 2015 se entendió oportuno realizar una nueva evaluación de la implementación de la educación sexual en todos los subsistemas implicados, que tomara en cuenta la visión de los operadores y los destinatarios del programa. Especialmente, porque la transición hacia la adultez requiere estar informado y equipado con competencias y conocimientos que permitan elegir alternativas responsables, tanto en nuestra vida social como sexual (UNESCO, 2010). Una educación efectiva en la temática de la sexualidad debe brindar información culturalmente relevante, científicamente rigurosa, y apropiada a la edad y al contexto específico de los estudiantes. Debe incluir, además, oportunidades estructuradas que les permitan explorar sus valores y actitudes, poniendo en práctica competencias esenciales para la toma de decisiones en relación con su vida sexual.

Diversos estudios demuestran que un programa académico efectivo de educación sexual debería tener la capacidad de: a) reducir la información incorrecta; b) aumentar el conocimiento y manejo de información correcta; c) clarificar y consolidar valores y actitudes positivas; $d$ ) fortalecer las competencias necesarias para tomar decisiones fundamentadas y la capacidad de actuar en función de ellas; e) mejorar las percepciones acerca de los grupos de pares y las normas sociales; y f) aumentar y mejorar la comunicación con padres, madres y otros adultos de confianza (UNESCO, 2010).

A pesar de lo antes mencionado y de que según la evaluación existe autonomía y flexibilidad para abordar los contenidos que propone el programa, algunos equipos de dirección 
ejercen controles que son vistos por los docentes como un riesgo para el amplio desarrollo de la formación en sexualidad. Las estudiantes mujeres manifiestan un mayor interés que los varones por las distintas temáticas, en especial por la diversidad sexual, la violencia doméstica e intrafamiliar, la violencia sexual, el género y los vínculos afectivos. Sin embargo, en el tema erotismo el interés de los varones supera ampliamente el de las mujeres. Asimismo, en Enseñanza Media el Programa de Educación Sexual no logra llegar de igual forma a Montevideo que al interior, y esto obstaculiza su implementación fuera de los límites de la capital (Peri, 2017).

En Uruguay, menos de un cuarto de la población es joven; en el resto de América Latina hay 52 jóvenes por cada 10 mayores de 65 años, en Uruguay hay 17. La tendencia demográfica marca un envejecimiento poblacional sostenido en el tiempo (Calvo, 2015). En cuanto a educación, prácticamente no se verifican casos de no asistencia al sistema educativo en las edades correspondientes al ciclo primario, por ende, es posible afirmar que existe una universalización de acceso, permanencia y egreso de la Enseñanza Primaria. Sin embargo, se constata un crecimiento de desvinculación del sistema educativo desde los 12 años en adelante. Igualmente, se debe señalar que la proporción de estudiantes que son excluidos del sistema educativo es claramente menor en 2018 que en 2006 (INEEd, 2019). Las estadísticas arrojan que los varones tienen una tasa de egreso significativamente menor que la de sus pares mujeres, y estas superan a los varones en la inmensa mayoría de indicadores educativos disponibles. En general, a las autoridades les preocupa que cuatro de cada diez jóvenes no logren culminar la educación obligatoria (último grado de Enseñanza Media). Por este motivo, se ha diseñado una serie de acciones que buscan la continuidad educativa. Además, esta realidad educativa muestra que la educación sexual formal no llega a la totalidad de adolescentes del país, sino que solo impacta en la biografía de los adolescentes escolarizados. Por lo cual, los agentes de socialización sexual de muchos adolescentes suelen ser exclusivamente la familia, el grupo de pares e Internet. A su vez, quienes se encuentran escolarizados reciben una educación sexual dispar, dependiendo de quién la dicta, el centro educativo donde se dicta y la ubicación geográfica de dicho centro, entre otros aspectos. Esta realidad repercute en la posibilidad de acceder a una educación sexual oportuna, de calidad y con equidad, que responda a las necesidades educativas reales de niños, niñas y adolescentes, y que acompañe sus procesos de apropiación subjetiva de derechos (Del Carmen, 2013).

Schenck (2014) plantea la coexistencia de tres tipos de discurso sobre diversidad sexual en el interior de las escuelas nacionales. El discurso excluyente, el tolerante y el de derechos. Ella afirma que el prevalente es el tolerante, y el de derechos se configura como un horizonte posible. Quizá esta conclusión, a la que arriba tras coordinar una investigación, es extrapolable para la educación en sexualidad en general. Asimismo, Gelpi (2019) en un artículo que presenta los resultados de un proyecto de investigación sobre la relación entre bullying homofóbico y exclusión escolar en el nivel de Ciclo Básico en liceos públicos y privados de la ciudad de Monte- 
video, concluye que la mayoría de los estudiantes han estado expuestos a una educación sexual heteronormativa, que reproduce desigualdades entre los estudiantes autoidentificados como LGBT y sus pares heterosexuales, todo lo cual atenta contra sus derechos humanos e incide en la promoción y preservación de la salud sexual de estos adolescentes. En suma, "se educa tanto a través de lo que se dice como de lo que se omite, de lo que se muestra como de lo que se margina, lo que se jerarquiza, legitima, devalúa o sanciona" (Bonder, 1994: 6).

La evidencia empírica disponible, nos conduce a la idea de que también se presenta una tensión entre el currículo explícito, lo oculto y el omitido. En ocasiones, el currículo explícito, vinculado a la educación sexual, se ve afectado por las creencias personales, por la moral sexual del propio referente de sexualidad y por sus experiencias personales, todo lo cual sustituye la evidencia científica en la transmisión de conocimiento. A veces, dicho currículo también se perjudica a causa del temor institucional ante la posibilidad de que los padres hagan reclamos por los contenidos que se abordan en el espacio aula. Esto se ha agravado en la región, especialmente a partir de la consolidación de los movimientos contra la ideología de género (Junqueira, 2018).

Los padres son los primeros responsables de la educación de la sexualidad de sus hijos. Tienen que ofrecerles explicaciones adecuadas a su edad para que adquieran el conocimiento y respeto de la propia sexualidad en un marco de confianza. Generalmente, se propone que es necesaria una explicación más que una prohibición, de lo contrario, los niños crecen desorientados, con dudas que resolverán con la persona menos indicada y con una información no del todo correcta. Pese a lo antes abordado, la escuela sigue teniendo un rol fundamental en la educación sexual, a modo de que los estudiantes no estén expuestos a un discurso único y poco riguroso sobre sexualidad. Sin embargo, se constata una falta de cooperación entre ambas instituciones (familia y escuela), porque en ocasiones la escuela representa una amenaza al mundo simbólico construido en el interior de los núcleos familiares de origen de los adolescentes. En esta línea, sería deseable una mayor corresponsabilidad y deconstruir la idea de que cuando se habla de sexualidad se está ante un tema sensible que debería ser exclusivo del mundo adulto (Del Carmen, 2013). Por otro lado, muchas veces los adolescentes pueden sentirse desconcertados por recibir información emitida por agentes de socialización sexual diametralmente opuesta o complementaria, pero a una distancia importante de sus propias cosmovisiones sobre el tema, por ende, se enfrentarán al desafío permanente de evaluar el contenido y construir sus propias verdades en esta materia.

Por estos motivos, aunque en la opinión pública la escuela sea vista como una institución en crisis y se hace referencia al vencimiento de los contenidos, la falta de formación de los docentes y la ausencia de interés del estudiantado, al sostener que la institución que se presentó durante décadas como "una maquinaria potente y eficaz, centro de irradiación de saberes, aparato clasificador de poblaciones, dispositivo modernizador, edificio público privilegiado, sím- 
bolo de la estatalidad, punto de encuentro y encierro de niños, jóvenes y docentes, máquina homogeneizadora, que pierde su poder para pasar a convertirse en el último reducto donde dichos elementos y procesos resisten, debilitados, a los embates de los tiempos actuales" (Pineau, 2007: 34), aún es posible reafirmar que, en ocasiones, en los distintos niveles educativos, pese a los cambios que ha habido, durante la trayectoria educativa de niños, niñas y adolescentes aún se dan pautas claras, diferenciadas y efectivas, por acción u omisión, en relación con lo que se espera socialmente de varones y mujeres, lo que puede limitar o potenciar la capacidad de performar el género y la sexualidad de los estudiantes (Schenck, 2014). La escuela, a partir de las normas de género, podría llegar a reforzar o deconstruir los modelos hegemónicos de masculinidad y feminidad, intervenir en la producción de subjetividad, de sexualidad masculina y femenina, y brindar información vinculada al ejercicio de esta.

Igualmente, las normas de género son dinámicas, varían dependiendo de la cultura y el contexto sociohistórico. Asimismo, los sujetos tienen capacidad de agenciamiento. Más allá de los efectos de los mensajes y prescripciones difundidas por los diferentes agentes de socialización (familia, escuela, Iglesia, medios de comunicación y grupos de pares), los sujetos podrían llegar a construir su propia masculinidad y/o feminidad con mayor o menor éxito, así como también, no tienen únicamente a la escuela como institución propicia para la socialización sexual (Ramos, Forrisi y Gelpi, 2015). Incluso, a veces la escuela también oficia como un espacio de crítica y reflexión, donde se ensayan alternativas al orden de género tradicional y al sistema patriarcal vigente, intentando deconstruir estereotipos y mandatos de género que producen desigualdades e introduciendo un discurso diferente al recibido dentro de los núcleos familiares de los estudiantes (Morgade, 2001).

De todos modos los cambios sociales se producen a una velocidad que la escuela es capaz de procesar parcialmente, y la realidad, cada vez más compleja, supera las capacidades de respuesta que demandan los distintos actores institucionales. Además, no es ni la misma escuela, ni son las mismas familias, ni son los mismos adolescentes, ni sus mismos problemas, y menos aún la misma sociedad. Es esperable que la institución esté en movimiento porque lo requiere para subsistir con cierta eficacia. Se intenta impulsar formal e informalmente una remodernización que contemple la dinámica vincular entre edificios del siglo XIX, profesores del siglo XX y estudiantes del siglo XXI. Algunas de las preguntas que pueden oficiar como disparadoras son: ¿qué escuela para qué adolescentes? ¿qué buscan los adolescentes hoy en la escuela? ¿qué tiene la escuela para ofrecerles? Además, como si fuese poco, en el cotidiano escolar se manifiesta una tensión entre legalidad y legitimidad, y pese a que Uruguay es visto por la comunidad internacional como un país de avanzada, caracterizado por su progresismo y agenda de derechos, tales atributos de momento se confirman más en un plano normativo que cultural, al menos en algunos contextos donde es una preocupación mantener el statu quo. 


\section{Etimología y surgimiento del concepto de adolescencia}

En el subsistema que se observa con detenimiento en este artículo, la población estudiantil suele transitar por la adolescencia. Por esta razón, se considera pertinente plantear algunas consideraciones sobre esta etapa del ciclo de vida. A lo largo de la historia, las sociedades y culturas conceptualizaron el ciclo de vida de diferentes formas y lo clasificaron en distintas etapas. La ciencia produjo conocimientos sobre las características y manifestaciones bio-psicosociales de los periodos evolutivos atribuyéndoles distintos significados (Ramos, 2015). La primera asociación moderna de la figura del joven hace referencia a la figura del estudiante y se ubica temporalmente en la revolución industrial. Es importante resaltar que adolescencia y juventud no significan lo mismo, configuran diferentes unidades de análisis, a pesar de que a veces se usan indistintamente. Toda categoría tiene su historia, y en la Antigua Grecia ya predominaba una mirada negativa sobre los jóvenes (Viñar, 2009).

Etimológicamente, el vocablo adolescencia proviene del latín adolescens, de la raíz adolescere, es decir "crecer". Igualmente, existe una construcción social del concepto, vinculada a "adolecer", como una etapa cargada de sufrimiento y padecimiento asociada a una crisis existencial (Amorín, 2010). También es posible entender la crisis como movimiento, cambio, lo que permitiría resignificar el concepto, ya que la adolescencia continúa siendo cargada de connotaciones exclusivamente negativas por un número no menor de académicos y técnicos.

La adolescencia, como producto de la sociedad occidental, llegó para cubrir las nuevas necesidades sociales, la mayoría de ellas, vinculadas con las relaciones de género y de clase social. Primero se adolescentizó a los niños de la clase burguesa y, a su vez, el destino de esos niños devenidos adolescentes sería distinto dependiendo del sexo (Fernández, 1998). Klein (2004) habla del espacio adolescente y la adolescencia como una creación de la modernidad, resultado de un desarrollo de procesos sociales, económicos y políticos.

La influencia del psicólogo estadounidense Stanley Hall en 1904, fue fundamental para que la adolescencia se estableciera como un nuevo campo de estudios dentro de la psicología evolutiva de la época. La perspectiva de Hall sobre la adolescencia era predominantemente negativa; en su tratado, se hace referencia al adolescente como transgresor, rebelde, y el autor usa frecuentemente palabras como tormentosa y dramática para describirla, trabaja sobre las tensiones y la aborda como un pasaje de transición hacia la adultez, lo que la dejaba en un inicio perdida en un entre, como algo inacabado entre la infancia y la adultez (Hall, 1904). Hasta finales de los años cincuenta, se sostuvo que la adolescencia era la cenicienta de la psicología, debido a las escasas producciones elaboradas por el campo $p s i$.

Barrán (1990) ubica la invención social del adolescente en Uruguay a finales del siglo XIX e inicios del XX. La creación de esta nueva figura social se rodearía de mitos, y se encomendó a actores como la policía, los médicos, maestros, sacerdotes, legisladores y las familias velar por el cuidado, control y disciplinamiento de estos debido a su tendencia hacia la impulsividad, la 
falta de represión sexual, la vagancia y la vandalización de los espacios públicos. Con los cambios que se fueron produciendo a lo largo del tiempo, fue recién a mediados del siglo XX que los adolescentes -en tanto grupo etario con características propias- se consolidaron en la escena social. Esto hace de la adolescencia una etapa y una categoría de reciente aparición en la historia de la humanidad, lo que se torna un desafío, incluso epistémico, en tanto campo de estudios, eje de políticas y categoría social.

A partir de los años setenta, gracias al fenómeno de la globalización —especialmente en su dimensión económica y cultural一, la adolescencia sufrió importantes cambios. El capitalismo y el culto al consumismo colocaron a la adolescencia en la industria cultural como un Target Group, diseñando un catálogo infinito de objetos culturales exclusivamente para adolescentes, inaugurando así, según Kancyper (2013: 49), una lógica de las diferencias. Él dirá que "la lógica del mercado es una lógica de las diferencias, un destino social que permite legitimar y discriminar individuos y grupos".

Estos hitos habilitaron nuevos sentidos para la adolescencia, la diversificaron a la vez que se buscaba la homogeneidad, se generaron tensiones entre los grupos de adolescentes y se instaló una jerarquización de las diferencias, profundizando paulatinamente aún más las ya existentes desigualdades sociales. De a poco, la adolescencia dejó de ser exclusivamente una etapa para convertirse en una imagen, en un producto cultural (Grosser, 2006).

Asimismo, los valores de la cultura del vértigo civilizatorio de las últimas décadas hicieron que existan nuevos modos de producción de subjetividad, entendida esta última como "pliegues de un conjunto de relaciones sociales, económicas y políticas que pautan formas específicas de socialización y de existencia" (De Brasi, 2005). Ante la crisis de varias instituciones de la modernidad, las identidades se fragilizaron $y$, entre otros aspectos, se pasó del culto a Dios al culto al cuerpo (Barrán, 1990). Los cuerpos entendidos ya no solo como máquinas de producción sino como un lugar de status y que otorga reconocimiento social, aunque con diferente destino y parámetros, dependiendo de la clase social de pertenencia del sujeto.

Actualmente, en el mejor de los escenarios, los adolescentes encuentran en los objetos culturales (investidos subjetivamente) y en su propia corporalidad, un reforzamiento a su propia identidad. Pero, para encontrar la aprobación de los pares, en cada contexto se deberá de tener ciertos objetos, determinado cuerpo que cumpla con los estrictos requisitos estéticos actuales y realizar algunas prácticas, si no, se correrá el riesgo de la discriminación, estigmatización y/o exclusión (Baudrillard, 1974). Según Weeks (1995: 90) en la contemporaneidad "el cuerpo es visto como la corte de enjuiciamiento final de lo que somos o en lo que nos podríamos volver".

La definición clásica de adolescencia de Amorín (2010), quien plantea a la misma como una categoría evolutiva que tiene derecho propio - evitando la mirada adultocéntrica que cree que lo importante es devenir en un adulto responsable como fin último-, y que está atravesada por aspectos psicosociales específicos (como la clase social a la que pertenece el adolescente, 
a la cultura y la sociedad en la que está inmerso) ya no es suficiente. Dada la compleja realidad social, Viñar (2009) afirma que lo más pertinente es hablar de adolescencias, en plural, a causa de los diversos modos de vivir, sentir y, a veces, de padecer de los adolescentes. Esto dependerá del contexto social, histórico, cultural, económico e inclusive político. Las características de cada adolescente "son producto de un cúmulo de interacciones entre lo individual y lo social en donde las categorías sexo, género, edad, nivel socioeconómico, lugar de procedencia y residencia, etnia y orientación sexual entre otros, se articulan de manera compleja" (Ramos, 2015: 17).

En relación con la sexualidad, en la adolescencia, como en otras etapas de la vida, esta cuenta con características, manifestaciones, necesidades, demandas propias y particulares. En este periodo, la transformación del cuerpo y el organismo, el autoerotismo, el inicio de las prácticas masturbatorias, el comienzo de la capacidad reproductiva y la iniciación sexual cobran vital importancia (López, 2015). A su vez, a los adolescentes no heterosexuales, se le suman otras particularidades relativas a su sexualidad, por ejemplo, el proceso de construcción de la identidad sexual y la necesidad de hacer la salida del closet (Ruiz, 2009).

Aunque existen adolescencias, hay procesos y duelos que se comparten en todos los contextos, que le permiten al propio sujeto reconocerse en tanto adolescente y que el entorno cercano lo reconozca como tal, por lo que, pese a los cambios sociales significativos que ha habido en los últimos tiempos, es posible decir que aún existe la adolescencia, que mantiene características de siempre, y que otras han mutado. Los procesos y duelos que se mantienen son: el duelo del cuerpo perdido, el duelo de los padres (desidealización de las figuras paternas), los ritos de iniciación, el rol que ocupan los grupos de pares, la necesidad de aprobación de sus pares -especialmente los del mismo sexo-y un nuevo ejercicio de la sexualidad (Kancyper, 1997; Viñar, 2009; Ramos, 2015). De todos modos, los ritos de iniciación también han variado con los años y han cobrado otros sentidos y significados, así como también se puede sostener que, tal vez, lo que más se ha alterado es la confrontación generacional a causa de la crisis de la institución familia por el inefectivo ejercicio parental, lo que afecta la conformación de la identidad de los adolescentes (Dolto, 1990; Viñar, 2009).

En la actualidad no existe unanimidad para definir la edad de comienzo y finalización de la adolescencia; depende del enfoque teórico de cada autor. En este escrito, se comparte la visión de la Organización Mundial de la Salud (OMS), la cual afirma que la adolescencia comienza a los 10 años y finaliza a los 19. Igualmente, dentro de esa franja, se ha conceptualizado una serie de subclasificaciones referidas a la adolescencia temprana, media y tardía, así como también se desarrolló el concepto de pubertad (Ramos, 2015). Por esta razón, la conceptualización de la(s) adolescencia(s) "es un campo en permanente construcción y objeto de debate, entre disciplinas e incluso dentro de los campos disciplinares que la convierten en objeto de estudio" (Ramos, 2015: 17).

Cuando se hace mención a los adolescentes uruguayos en la contemporaneidad, es posible identificar algunas características comunes a este grupo etario: nacieron en democracia, 
crecieron con la globalización, la crisis de la modernidad, la revolución científica tecnológica, la era de la informática y las nuevas tecnologías, el auge de las redes sociales online, las crisis económicas y de las funciones protectoras del Estado-nación y sus instituciones, los fuertes cambios en las familias y la subjetividad, ejercen activamente su sexualidad a edades más tempranas que antiguas generaciones, muchos rechazan las etiquetas sexuales y fueron testigos de la revolución del movimiento feminista y de la diversidad sexual.

Un aspecto negativo es que los adolescentes en nuestro país aún continúan teniendo en la sociedad un lugar de menor poder y representatividad que las personas adultas. Asimismo, "al interior del colectivo adolescente existe gran inequidad respecto a las posibilidades de ejercer los derechos humanos, incluidos los derechos sexuales y reproductivos" (Ramos, 2015: 19). Dicha inequidad generacional se asocia a la desigualdad de acceso a oportunidades, derechos, recursos, bienes y servicios entre los grupos de edad en un mismo contexto sociohistórico (Ramos, 2015).

\section{Confrontación generacional y cuestiones de género: la lucha activa dentro y fuera de las escuelas}

Primeramente, es pertinente introducir el concepto generación, el cual remite a la edad, pero procesada por la cultura y la historia. Como lo indica Margulis y Urresti (1998: 6), se es generalmente solidario con los códigos culturales incorporados durante la socialización, "hay afinidades con otros miembros de la misma generación con los que se comparten espacios sociales y, por ende, desde esa perseverancia generacional, se entra en contradicción y en desencuentro con las cohortes generacionales siguientes".

Desde hace algunas décadas, pero particularmente en los últimos años y en contextos socioeconómicos específicos, el movimiento feminista y LGBT le han brindado al menos a un grupo de adolescentes nuevos elementos para sus procesos de construcción identitaria.

La confrontación generacional, como se insinuó anteriormente, es un proceso esencial para la adquisición de la identidad. Su condición primera es la presencia de otro como una alteridad, ni blanda ni arbitraria, que posibilita la tensión de la diferencia entre los opuestos, admitiendo ambas partes que ser oponente no es equivalente a ser enemigo. Sin este arco de tensiones "se paraliza la dialéctica de las identificaciones-desidentificaciones y reidentificaciones que se despliega durante toda la vida pero de un modo privilegiado durante el período de la adolescencia" (Kancyper, 2004: 93). Esta etapa se caracteriza por el definitivo desprendimiento mental de los padres a través de la superación del complejo de Edipo y de la terminación del desarrollo sexual, por lo que se puede relacionar directamente con los procesos de subjetivación que llevan adelante los adolescentes.

Es estimulante que la adolescencia esté activa y haga oír su voz, pero los esfuerzos adolescentes que hoy se hacen sentir en todo el mundo deben ser enfrentados, deben cobrar realidad 
gracias a un acto de confrontación. Esta debe ser personal. Los adultos son necesarios para que los adolescentes tengan vida y vivacidad, que los adolescentes modifiquen la sociedad y enseñen a los adultos a ver el mundo de una manera nueva — en la medida de lo posible, con los lentes violeta puestos-; pero que allí donde esté presente el desafío de un joven en crecimiento, haya un adulto dispuesto a enfrentarlo (Winnicott, 1972).

En este sentido, en la actualidad, las cuestiones relativas al género y a la sexualidad ocupan un rol preponderante en el proceso de la confrontación generacional entre adolescentes y adultos. Existen matices entre ambas generaciones en cuanto a las creencias de género, las normas de género, los mensajes de género, los estereotipos de género, los modelos disponibles de masculinidad y feminidad, y los permisos y prohibiciones en materia de sexualidad que los sujetos tienen siempre generizados. Además, la noción de género es estructurante del psiquismo e incluso preexiste al proceso de diferenciación sexual característico de la angustia de castración en la fase fálica del desarrollo psicosexual (Laplanche, 2006). Este novedoso modo de transitar la confrontación generacional, atravesada más que nunca por cuestiones de género, en gran parte ha sido habilitado por los aportes del movimiento feminista y de la diversidad sexual.

El feminismo constituye una cultura que, en su globalidad, es crítica de un sujeto social -las mujeres-, de la sociedad y la cultura dominante, pero es mucho más; es afirmación intelectual, teórica y jurídica de concepciones del mundo, modificación de hechos, relaciones e instituciones; es aprendizaje e invención de nuevos vínculos, afectos, lenguajes y normas; se plasma en una ética y se expresa en formas de comportamiento nuevas tanto de mujeres como hombres (Lagarde, 2012: 461).

Esta realidad introduce a la discusión la necesidad de que quizá aún exista un orden de género tradicional, expresiones de masculinidad y feminidad hegemónicas y subjetividades más conservadoras en nuestras sociedades, porque dicha existencia termina orientando a los adolescentes a tomar contacto con qué tipo de adultos desean y pueden ser. Se insiste en el "pueden ser", porque quizá hay muchos adolescentes que a nivel de creencias y de discurso están afines con una sociedad más igualitaria, pero quedan rehenes de barreras afectivas y cognitivas que no les permiten realizar un cambio comportamental relativo a las cuestiones de género en sus vidas cotidianas. En suma, una cosa es la toma de conciencia y otra el cambio comportamental que lleva al cambio social. Asimismo, si se realiza un análisis atravesado por clase social de pertenencia, es probable que producir ciertas narrativas y realizar determinadas prácticas sociales y políticas en algunas ciudades, pueblos, localidades y barrios del país sea aún más complicado, porque les deja expuestos a ser objetos de violencia y estigmatización por parte de sus pares y otros adultos referentes, por ende, no todos pueden realizar estos procesos de tránsito genérico hacia una mayor libertad (sexual). En este artículo, se piensa el concepto de tránsito genérico con base en la producción de Parra (2019), ella plantea que no todo tránsito genérico tiene como punto de partida la cisgeneridad y como punto de llegada la transgeneridad, 
sino que muchas personas transitan dentro de su propia identidad de género al problematizar, revisar, deconstruir y transformar algunas creencias, actitudes y comportamientos vinculados al género y la sexualidad.

De todos modos, se debe resaltar que, en general, en estos contextos posibles emergen nuevas formas de organización y de resistencia al sistema sexo-género dominante y a las estructuras institucionales patriarcales y heteronormativas. A nivel educativo, en la actualidad, muchos adolescentes incluso discuten los (no) contenidos que reciben dentro del espacio aula, interpelan los discursos y prácticas de diferentes actores educativos que refuerzan la heteronormatividad y la cisgeneridad. Todo lo cual, promueve la exigencia de legitimidad de nuevas formas de ser y estar dentro de los edificios escolares en el marco de una educación laica. A su vez, esto se traduce en condiciones propias para el ejercicio de ciudadanía por parte de un grupo poblacional históricamente silenciado e invisibilizado en cuanto a su potencialidad política. Un grupo de adolescentes contemporáneos se aleja de esa asignación de ser la generación "nini" o de ser "ciudadanos anestesiados" que solo se expresan a través de las redes sociales online; este grupo de adolescentes ponen el cuerpo en el espacio público en el marco del retorno de los gobiernos neoliberales y neoconservadores en la región, de hecho, en ocasiones su lucha no es conquistar necesariamente nuevos derechos, sino más bien se trata de defender los ya consagrados y actualmente amenazados.

Las prácticas antes mencionadas pueden asemejarse al concepto de sororidad, el cual parte de un esfuerzo por desestructurar la cultura y la ideología de la feminidad que encarna cada mujer, como un proceso que se inicia en la amistad/enemistad de las mujeres y avanza en la amistad de las amigas, en busca de tiempos nuevos, de nuevas identidades. "La sororidad como superación de la relación más opresiva de todas, que es la relación real, simbólica, imaginaria y fantástica, que mantiene un lazo desigual y asimétrico entre las mujeres" (Lagarde, 2012: 494). Igualmente, este tipo de prácticas no son realizadas únicamente por mujeres, un grupo de varones también comienza a problematizar sus propias identidades genéricas (Burin y Meler, 2009).

Este accionar permite resignificar la construcción social del "ser ciudadano", la cual guarda relación histórica y directa con el sistema patriarcal, porque se trata de una categoría creada desde el imaginario de lo masculino. En este caso, las adolescentes mujeres se han mostrado muy activas en su militancia, combatiendo que las voces públicas generalmente sean la de los varones y los silencios privados generalmente de las mujeres (Astelarra, 1990). De todos modos, es pertinente recordar que existen restricciones de orden sociocultural, político y económico que subsisten, e impiden especialmente que gran parte de las mujeres puedan ejercer su ciudadanía activamente.

Entendemos por ciudadanía un concepto que sintetiza la relación entre el Estado y las personas. "Una construcción con múltiples niveles, el cual se aplica a la pertenencia de la población a una variedad de colectividades locales, étnicas, nacionales y transnacionales" (Yuval-Davis, 
1996: 2). 'Las colectividades y 'comunidades' son constructos ideológicos y materiales cuyas fronteras, estructuras y normas son resultado de procesos constantes de luchas y negociaciones, o desarrollos sociales más generales (Yuval-Davis ,1996: 6). Esta reflexión brinda elementos para pensar los procesos de identificación personal y colectiva con la lucha del movimiento feminista. En esta línea, es frecuente: a) la existencia de comisiones de género dentro de los centros estudiantiles de las instituciones de enseñanza media; $b$ ) la convocatoria abierta para concurrir a marchas que defienden los derechos de las mujeres y de la población LGBTIQ+; c) prácticas de solidaridad de género organizadas ante situaciones de discriminación de género dentro de los edificios escolares; y d) la toma de liceos ante casos de acoso sexual realizado por docentes hacia estudiantes, predominantemente mujeres.

Ante la inacción del mundo adulto o el temor de los docentes y autoridades de trabajar ciertos temas en aula, los adolescentes devienen sujetos políticos activos, aparecen en la escena pública y ponen sus cuerpos para denunciar desigualdades estructurales y para promover el cambio social. Uno de los hitos contemporáneos más significativos es la reivindicación del uso de pañuelos en tanto símbolos culturales. En Argentina se visualizó el uso del pañuelo verde a favor del aborto legal, seguro y gratuito. En Uruguay se popularizó el uso del pañuelo amarillo a favor de la ley integral trans. Los pañuelos suelen colocarse en las mochilas, cuello o muñecas de los estudiantes tanto varones como mujeres, son visibles al resto de la comunidad, ingresan a las escuelas con ellos, marcan una posición ética y política relativa a la agenda de género y combaten los procesos históricos de silenciamiento e invisibilización de la diversidad sexogenérica dentro de los edificios escolares, desestabilizando el curriculum oculto, no sin correr el riesgo de ser víctimas de estereotipos, prejuicios sociales, sexuales y de género, discriminación, bullying y exclusión.

Quizá la identificación con este símbolo cultural aporta a la tarea de deconstruir la asociación directa de género igual a problemas de mujeres $y$, al mismo tiempo, deja en evidencia la convivencia de múltiples subjetividades singulares y colectivas al interior de las escuelas. Por otro lado, la escuela es vista como un espacio de negociación de sentidos en relación con el género y la sexualidad, donde se hace visible una lucha entre subjetividades que defienden el sistema dominante y otro grupo de subjetividades contrahegemónicas que promueven relaciones de género más igualitarias. Tal vez una de las claves sea reflexionar sobre el rol que debe asumir la educación sexual formal en todo esto.

En suma, un pañuelo es una prenda o accesorio que a través de los siglos logró revertir su carga simbólica de fragilidad (relacionado con las lágrimas, despedidas o incluso el perfume que le dio un sentido romántico), para concentrar en su escasa superficie una fuerte capacidad de representación. La socióloga argentina Zambrini (2010) piensa a los pañuelos como marcas culturales inscritas en el cuerpo. Esto refuerza la idea de que es imposible pensar el género y la sexualidad excluyendo los cuerpos (Glocer Fiorini, 2016), cuerpos de adolescentes sexuados 
y politizados, con voluntad de ejercer ciudadanía activamente, a veces desafiando a los guiones sociales y sexuales pensados por la propia escuela como destino para este colectivo. Es fundamental señalar que no se trata de subjetividades sedentarias, ni de cuerpos dóciles; en este artículo se intentó presentar a una generación de adolescentes que tienen la convicción ideológica de que la revolución, finalmente, será feminista o no será. Probablemente una de las preguntas que se pueden formular sea si la escuela quiere y puede acompañarlos como ellos desean, en ese tránsito hacia la adultez, el cual tiene predominantemente una dimensión intrapsíquica, otra interpersonal y una última, cultural, que implica incorporar herramientas para la construcción de autonomía, para apropiarse subjetivamente de sus derechos y para devenir ciudadanos críticos con capacidad de incidencia en la esfera pública.

\section{Consideraciones finales}

En este artículo se presentó una breve historización del sistema educativo formal uruguayo que retomó sus características, objetivos y estructura; se abordó el proceso de diseño, implementación y evaluación del Programa de Educación Sexual de la Administración Nacional de Educación Pública; se retomaron los principales desafíos contemporáneos en cuanto a la definición del currículo, su funcionamiento y las resistencias que impiden que este programa se legitime en la comunidad educativa como otro saber más; se ahondó en el rol de la institución familia en el marco de la educación en sexualidad de los adolescentes; y se explicitó la necesidad de una mayor corresponsabilidad entre la institución familia y la institución educativa.

Es posible concluir que la educación sexual formal no llega a la totalidad de adolescentes del país sino que solo incide en la biografía de los adolescentes escolarizados. Por lo cual, los agentes de socialización sexual de muchos adolescentes suelen ser exclusivamente la familia, el grupo de pares e Internet, con todo lo que eso significa. A su vez, quienes se encuentran escolarizados reciben una educación sexual dispar, dependiendo de quién la dicta, el centro educativo donde se dicta y la ubicación geográfica de dicho centro, entre otros aspectos. Esta realidad repercute en la posibilidad de acceder a una educación sexual oportuna, de calidad y con equidad, que responda a las necesidades educativas reales de niños, niñas y adolescentes (Del Carmen, 2013).

Además, en ocasiones, el currículo explícito vinculado a la educación sexual se ve afectado por las creencias y experiencias personales del referente en sexualidad, así como también por su propia moral sexual, todo lo cual sustituye a la evidencia científica en la transmisión de conocimiento. Este escenario produce que muchas veces los adolescentes puedan sentirse desconcertados por recibir información emitida por agentes de socialización sexual diametralmente opuesta o complementaria, pero a una distancia importante de sus propias cosmovisiones sobre el tema, por ende, se enfrentarán al desafío permanente de evaluar el contenido y construir sus propias verdades en esta materia. 
Asimismo, se desarrollaron conceptualizaciones teóricas relativas a la adolescencia en tanto etapa del ciclo de vida, deteniéndose especialmente a reflexionar sobre los procesos de confrontación generacional entre los adolescentes y sus referentes adultos. Desde hace algunas décadas, pero particularmente en los últimos años y en contextos socioeconómicos específicos, el movimiento feminista y LGBT le han brindado al menos a un grupo de adolescentes nuevos elementos para sus procesos de construcción identitaria. En ciertos contextos posibles, emergen nuevas formas de organización y de resistencia al sistema sexo-género dominante y a las estructuras institucionales patriarcales y heteronormativas, promoviéndose la exigencia de legitimidad de nuevas formas de ser y estar dentro de los edificios escolares, en el marco de una educación laica. Esto se traduce en condiciones propias para el ejercicio de ciudadanía por parte de un grupo poblacional históricamente silenciado e invisibilizado en cuanto a su potencialidad política.

Los adolescentes devienen sujetos políticos activos, aparecen en la escena pública y ponen sus cuerpos para denunciar desigualdades estructurales y para promover el cambio social. Uno de los hitos contemporáneos más significativos es la reivindicación del uso de pañuelos en tanto símbolos culturales. Los pañuelos suelen colocarse en las mochilas, cuello o muñecas de los estudiantes, tanto varones como mujeres, son visibles al resto de la comunidad, ingresan a las escuelas con ellos, marcan una posición ética y política relativa a la agenda de género y combaten los procesos históricos de silenciamiento e invisibilización de la diversidad sexo-genérica dentro de los edificios escolares, desestabilizando el curriculum oculto, no sin correr el riesgo de ser víctimas de estereotipos, prejuicios sociales, sexuales y de género, discriminación, bullying y exclusión.

Quizá la identificación con este símbolo cultural aporta a la tarea de deconstruir la asociación directa de género igual a problemas de mujeres y, al mismo tiempo, deja en evidencia la convivencia de múltiples subjetividades singulares y colectivas en el interior de las escuelas. Por otro lado, la escuela es vista como un espacio de negociación de sentidos en relación con el género y la sexualidad, donde se hace visible una lucha entre subjetividades que defienden el sistema dominante y otro grupo de subjetividades contrahegemónicas que promueven relaciones de género más igualitarias.

Esto refuerza la idea de que es imposible pensar el género y la sexualidad excluyendo los cuerpos (Glocer Fiorini, 2016), cuerpos de adolescentes sexuados y politizados, con voluntad de ejercer ciudadanía activamente, a veces desafiando a los guiones sociales y sexuales pensados por la propia escuela como destino para este colectivo. Es fundamental señalar que no se trata de subjetividades sedentarias, ni de cuerpos dóciles; en este artículo se intenta aportar al ejercicio de imaginar la educación sexual que se precisa para potenciar las capacidades de los adolescentes, especialmente porque se trata de una generación que tiene la convicción ideológica de que la revolución será feminista o no será. 


\section{Referencias bibliográficas}

Abero, B. (2015). La educación sexual como política pública en Uruguay. En López, A. (coord.). Adolescentes y sexualidad. Investigación, acciones y política pública en Uruguay (2005-2014). Montevideo: UNFPA.

Amorín, D. (2010). Apuntes para una posible psicología evolutiva. Tomo I. Montevideo: Ediciones Psicolibros Waslala.

Astelarra, J. (1990). Participación política de las mujeres. Madrid: Centro de Investigaciones Sociológicas.

Barrán, J. P. (1990). Historia de la sensibilidad en el Uruguay. Tomo 2: El disciplinamiento (18691920). Montevideo: Ediciones de la Banda Oriental.

Baudrillard, J. (1974). La sociedad de consumo. Sus mitos, sus estructuras. Madrid: Siglo XXI Editores.

Bayce, R. (1987). Sistema educativo y crisis nacional. Montevideo: Facultad de Ciencias Sociales.

Bonder, G. (1994). Mujer y educación en América Latina: Hacia la igualdad de oportunidades. Revista Iberoamericana de Educación, (6), 9-48.

Britzman, D. (1996). O que é essa coisa chamada amor: identidade homossexual, educação e currículo. Educação e Realidade, 21(1), 71-96.

Burin, M.; I. Meler (2009). Varones. Género y subjetividad masculina. Buenos Aires: Librería de Mujeres Editoras.

Calvo, J. (2015). Atlas sociodemográfico y de la desigualdad del Uruguay. Jóvenes en Uruguay: demografía, educación, mercado laboral y emancipación. Montevideo: INE.

Darré, S. (2005). Políticas de género y discurso pedagógico. La educación sexual en el Uruguay del siglo XX. Montevideo: Ediciones Trilce.

De Brasi, J. C. (2005). Dimensiones de la grupalidad. Montevideo: Editorial Multiplicidades.

Del Carmen, V. (2013). Educación de la sexualidad en el contexto familiar y escolar. Educere, $17(58), 429-435$.

Dolto, F. (1990). La causa de los adolescentes. Barcelona: Seix Barral.

Elizalde, S. (2009). Normalizar ante todo. Ideologías prácticas sobre la identidad sexual y de género de los/as jóvenes en la dinámica de las instituciones orientadas a la juventud. Revista Argentina de Estudios de Juventud, 1(1), 1-11.

Errandonea, G. (2014). A 140 años de la educación del pueblo: Aportes para la reflexión sobre la educación en Uruguay. Tomo I. Educación Pre primaria, Primaria y Media. Montevideo: MEC.

Fernández, A. (1998). La sexualidad atrapada de la señorita maestra. Una lectura psicopedagógica del ser mujer, la corporalidad y el aprendizaje. Buenos Aires: Ediciones Nueva Visión.

Filgueira, F.; R. Kaztman (2001). Panorama de la infancia y la familia en Uruguay. Montevideo: Universidad Católica del Uruguay. 
Fondo de Población de las Naciones Unidas (UNFPA). (2014). La educación sexual en Uruguay, su aporte para el ejercicio de ciudadanía. Población y Políticas Públicas: Apuntes para el debate, (5). Montevideo: UNFPA.

Foucault, M. (1998). Historia de la sexualidad. Vol. I: La voluntad de saber. Madrid: Siglo XXI Editores.

Freitas, P. (2016). Vestidos en el aula. Guía educativa sobre diversidad afectivo sexual. Montevideo: Llamale $\mathrm{H}$.

Gelpi, G. (2019). Ser víctima de bullying homofóbico en Uruguay. Las voces de varones adolescentes de la ciudad de Montevideo. Revista de la Escuela de Ciencias de la Educación, 1(14), 65-82.

Glocer Fiorini, L. (2016). Cuerpo, género y sexo: Una relación paradojal. Revista Uruguaya de Psicoanálisis, 123, 104-113.

Grosser, K. (2006). La juventud como mercancía y el lugar de lo adolescente en la lógica cultural del capitalismo tardío. Revista Actualidades Investigativas en Educación, 6(2), 2-21.

Hall, S. (1904). Adolescence: It's a Psychology and its Relations to Psychology, Anthopology, Sociology, Sex, Crime, Religion, and Education. Nueva York: D. Appleton and Company.

Instituto Nacional de Evaluación Educativa (INEEd). (2019). Informe sobre el estado de la educación en Uruguay 2017-2018. Montevideo: INEEd.

Junqueira, R. D. (2013). Pedagogia do armário. A normatividade em ação. Revista Retratos da Escola, 7(13), 481-498.

Junqueira, R. D. (2018). Ideología de gênero: Um dispositivo retórico de uma ofensiva antifeminista. En Ferreira, A.; E. Ferreira; M. Santana (coords.). Gênero e sexualidades: Entre invenções e desarticulações. Aracajú: Editora IFS.

Kancyper, L. (2013). Adolescencia: El fin de la ingenuidad. Querencia, Revista de Psicoanálisis, 14(2), 45-55.

Kancyper, L. (2004). Adolescencia y confrontación generacional: Los afectos y el poder. Revista APPIA, 15, 92-114.

Kancyper, L. (1997). La confrontación generacional. Estudio psicoanalítico. Buenos Aires: Paidós. Klein, A. (2004). Adolescencia: Un puzzle sin modelo para armar. Montevideo: Ed. Psicolibros. Lagarde, M. (2012). El feminismo en mi vida: Hitos, claves y topías. Ciudad de México: INMUJERES DF.

Laplanche, J. (2006). El género, el sexo, lo sexual. Revista ALTER, 2, 1-15.

Ley Núm. 18. 437. (2009). Ley General de Educación. Montevideo: IMPO.

López, A. (coord.). (2015). Adolescentes y sexualidad. Investigación, acciones y política pública en Uruguay (2005-2014). Montevideo: UNFPA.

Louro, G. L. (2000). O corpo educado: Pedagogias da sexualidade. Belo Horizonte: Autêntica. 
Margulis, M.; M. Urresti (1998). La construcción social de la condición de juventud. Buenos Aires: Espasa Calpe.

Marrero, A.; N. Mallada (2009). La Universidad transformadora. Montevideo: Facultad de Ciencias Sociales.

Marrero, A.; N. Mallada; G. Cafferatta (2008). El bachillerato uruguayo y su lugar como tránsito hacia el mundo universitario y del mundo del trabajo. Montevideo: Facultad de Ciencias Sociales.

Marrero, A. (2003). Educación Secundaria: Desigualdad, estructuración social y vida cotidiana en los liceos públicos. Montevideo: Facultad de Ciencias Sociales.

Morgade, G. (2006). Sexualidad y prevención: Discursos sexistas y heteronormativos en la escuela media. Revista del Instituto de Ciencias de la Educación, 24, 1-20.

Morgade, G. (2001). Aprender a ser mujer, aprender a ser varón. Buenos Aires: Ediciones Novedades Educativas.

Nahum, B. (2008). Historia de Educación Secundaria 1935-2008. Montevideo: ANEP/CODICEN/CES/ Plan General de Obra.

Organización de las Naciones Unidas para la Educación, la Ciencia y la Cultura (UNESCO) (2010). Orientaciones técnicas internacionales sobre educación en sexualidad. Un enfoque basado en evidencia orientado a escuelas, docentes y educadores de la salud. París: UNESCO.

Ovejas Negras y GLSEN (2016). Encuesta Nacional de Clima Escolar en Uruguay 2016. Experiencias de niños, niñas y adolescentes lesbianas, gays, bisexuales y trans en establecimientos educativos. Montevideo: Ovejas Negras.

Parra, N. (2019). Diversidad de género en las infancias. Las Palmas: Fundación Universitaria de Las Palmas.

Peri, A. (2017). Evaluación del Programa de Educación Sexual. Montevideo: ANEP/UNFPA.

Pineau, P. (2007). Algunas ideas sobre el triunfo pasado, la crisis actual y las posibilidades futuras de la forma escolar. En Baquero, R.; G. Diker; G. Frigerio (coords.). Las formas de lo escolar. Buenos Aires: Del Estante Editorial.

Ramos, V. (2015). Consideraciones conceptuales: Adolescencia, sexualidad y derechos. En López, A. (coord.). Adolescentes y sexualidad. Investigación, acciones y política pública en Uruguay (2005-2014). Montevideo: UNFPA.

Ramos, V.; F. Forrisi; G. Gelpi (2015). Nociones básicas sobre sexualidad, género y diversidad: un lenguaje en común. En López, P.; F. Forrisi; G. Gelpi (coords.). Salud y diversidad sexual. Guía para profesionales de la salud. Montevideo: MSP.

Ruiz, S. (2009). Diversidad sexual en las aulas. Evitar el bullying o acoso homofóbico. Badajoz: Cuadernos Plural.

Schenck, M. (2014). Sacando la violencia heteronormativa del armario: Docentes LGB y discriminación. En Sempol, D. (coord.). De silencios y otras violencias. Políticas públicas, regulaciones discriminatorias y diversidad sexual. Montevideo: MIDES. 
Sempol, D. (coord.) (2013). Políticas públicas y diversidad sexual. Análisis de la heteronormatividad en la vida de las personas y las instituciones. Montevideo: MIDES.

Sempol, D.; F. Graña (2012). Salvar la patria y a la familia. Dictadura, disidencia sexual y silencio. En González, S.; M. Russo (coords.). Las Laurencias. Violencia sexual y de género en el terrorismo de Estado uruguayo. Montevideo: Trilce.

Silveira, N. (2015). Con la soga al cuello. El imaginario social de la violencia en docentes de enseñanza pública. Montevideo: CSIC.

Viñar, M. (2009). Mundos adolescentes y vértigo civilizatorio. Montevideo: Ediciones Trilce.

Viscardi, N. (2008). Violencia en las aulas. Práctica educativa y conflicto escolar y exclusión social. En Paternain, R.; R. Sanseviero (coords.). Violencia, inseguridad y miedos en el Uruguay. ¿Qué tienen para decir las ciencias sociales? Montevideo: FESUR.

Viscardi, N. (2003). Enfrentando la violencia en las escuelas: Un informe de Uruguay. En Abramovay, M. (coord.). Violência na escola. América Latina e Caribe. Brasilia: UNESCO.

Weeks, J. (1995). Invented Moralities: Sexual Values in an Age of Uncertainty. Nueva York: Columbia University Press.

Winnicott, D. (1972). Realidad y juego. Buenos Aires: Granica.

Yuval-Davis, N. (1996). Mujeres, ciudadanía y diferencia. Londres: Universidad de Greenwich.

Zambrini, L. (2010). Modos de vestir e identidades de género: Reflexiones sobre las marcas culturales en el cuerpo. Revista Nomadías, (11), 130-149. 